\title{
Immunomodulatory Second-Line Therapies for Immune Thrombocytopenia
}

\author{
Michele P. Lambert ${ }^{1,2}$ \\ ${ }^{1}$ Division of Hematology, The Children's Hospital of Philadelphia, \\ Philadelphia, Pennsylvania, United States \\ 2 Department of Pediatrics, Perelman School of Medicine at the \\ University of Pennsylvania, Philadelphia, Pennsylvania, United States
}

Address for correspondence Michele P. Lambert, MD, MSTR, Division of Hematology, The Children's Hospital of Philadelphia, 3615 Civic Center Blvd ARC 316G, Philadelphia, PA 19104-4399, United States (e-mail: lambertm@email.chop.edu).

Hämostaseologie 2019;39:266-271.

\author{
Abstract \\ Keywords \\ - autoimmune disease \\ - platelet \\ - ITP \\ - treatment
}

Management of immune thrombocytopenia (ITP) is complex requiring communication between patients and caregivers to establish a mutual understanding of the impact of the patient's disease on quality of life, the current symptoms and risk of morbidity/ mortality and the goals of therapy. The currently available second-line therapies for ITP provide potential for management of thrombocytopenia and bleeding symptoms with medical therapy or surgical intervention potentially offering long-term remission. All therapies are associated with potential side effects and necessary monitoring or modifications/risks and careful discussion of these is necessary to determine the optimal therapy for each patient. This review covers second-line therapies for ITP and discusses the currently available information on immunomodulatory second-line treatments for ITP.

\section{Introduction}

Immune thrombocytopenia (ITP) is an autoimmune disease defined by isolated thrombocytopenia (platelets $<100 \times 10^{9} / \mathrm{L}$ ). Treatment paradigms for ITP have assumed at the onset a fundamental difference between paediatric and adult ITP describing paediatric ITP to be generally self-limited disease with abrupt onset and profound thrombocytopenia while adult ITP is considered a more chronic disease with more insidious onset, moderate thrombocytopenia and higher bleeding risk with platelet counts $<30 \times 10^{9} / \mathrm{L}$ and increased morbidity and mortality. ${ }^{1,2}$ Recent data suggest that presenting platelet counts are not that different and that the likelihood of bleeding, overall, with platelets of $<20 \times 10^{9} / \mathrm{L}$ is low in most patient populations. ${ }^{3}$ This study, prospectively following outcomes in approximately 3,360 children and 420 adults with ITP from several countries over several years, also demonstrated a higher than expected remission rate in the adult population ( $45 \%$ at 6 months), although the majority of adults were young adults (250/420 were young adults). Late remission was similar in children

received

September 6, 2018

accepted after revision

February 14, 2019

and adults (approximately 30\% at 12 and 24 months of follow-up). ${ }^{3}$

At the time of the publication of the last guidelines (20092011), ${ }^{4,5}$ there was little data available on the long-term safety and efficacy of some of the second-line therapies for ITP, in particular for the thrombopoietin receptor agonists (TPO-RAs), such as eltrombopag and romiplostim which are approved for adults and children and lusutrombopag and avatrombopag, which are approved for adults with chronic liver disease. These medications are covered in a separate review in this issue and are not discussed here, but have changed the landscape of second-line therapy for ITP. This review will focus on nonTPO-RA second-line therapy for ITP in patients requiring treatment. Generally, treatment is warranted for adult patients with platelet counts $<30 \times 10^{9} / \mathrm{L}$ (although data suggest this number may be higher than necessary), or in patients at risk for bleeding. ${ }^{4}$ Often, patient quality of life determines need for therapy, irrespective of bleeding symptoms in both paediatric and adult populations. ${ }^{6,7}$ Patients that fail first-line therapy often require second-line therapy, and may progress through multiple second-line therapies. (c) 2019 Georg Thieme Verlag KG
Stuttgart · New York
DOI https://doi.org/ 10.1055/s-0039-1684032. ISSN 0720-9355. 


\section{Splenectomy}

The first splenectomy for ITP was performed in $1916^{8}$ and for decades, surgical splenectomy was the second-line treatment of choice because this is an effective treatment for steroid-refractory or dependent ITP with 50 to $70 \%$ of patients achieving a durable remission. ${ }^{9,10}$ However, recent data suggest that $<25 \%$ of patients with ITP undergo splenectomy, ${ }^{11}$ despite these excellent durable response rates $^{12,13}$ and decades of experience. Declining splenectomy is likely in part due to the risk of infection (5- to 30-fold increase in the first 90 days and 1- to 3-fold life-long increased risk of invasive bacterial infection and sepsis) and emerging data on risk of thrombosis as well as reports of pulmonary hypertension combined with immediate postoperative complications in the setting of available effective medical therapy. Most studies examining risk of mortality in splenectomy do not stratify by indication; however, Thai and colleagues examined the long-term complications of splenectomy in ITP patients in particular, ${ }^{14}$ finding that in 93 patients with ITP, $17 \%$ of patients had early post-operative complications including haemorrhage, infection and venous thromboembolism (VTE). After a median follow-up of 192 months (range: $0.5-528$ ), 52\% had a sustained response and $80 \%$ were alive. The rate of VTE in this study was $16 \%$ in the splenectomy group versus $2 \%$ in the control group (consisting of patients with ITP who had not undergone splenectomy matched for date of diagnosis, age and gender). ${ }^{14}$ These included both immediate post-operative VTE and VTE 10, 20 and 30 years post-splenectomy. A second recent long-term follow-up study of 174 adult patients who underwent splenectomy had a $2.9 \%$ rate of VTE in their cohort. $^{15}$ The smaller study also suggested an increased risk of cardiovascular events compared with control patients (12 vs. $5 \%$ ), although this did not reach statistical significance $(p=0.143) .{ }^{14}$ The rates of infection were not significantly different between splenectomy and control; however, the rate of bacterial infection was higher in the post-splenectomy group and these infections were more likely to result in hospitalization (all of the post-splenectomy patients) with an increased risk of sepsis (19\%) with three fatalities (vs. 0 for the control group). ${ }^{14}$ Other studies have suggested an overall risk of mortality from overwhelming post-splenectomy infection of 0.73 per 1,000 patient years. ${ }^{13}$ These data support the overall assessment that splenectomy is relatively safe, but not without risk or potential long-term complications. Generally, splenectomy is deferred at least 12 months if possible (although it is still the treatment for fulminant ITP refractory to intravenous immunoglobulin/corticosteroids especially if there is poor response to TPO-RA). ${ }^{6}$ Patients who undergo splenectomy should be vaccinated for pneumococcus, Haemophilus influenzae B and meningococcus C prior to splenectomy whenever possible. ${ }^{16}$ Because there is very little data to compare long-term outcome of splenectomy with long-term medical treatment, evidence-based recommendations are lacking and decisions are often based on patient and physician discussion and mutual discussion of patient factors, preferences and goals of therapy. ${ }^{6,17,18}$
Patients who prefer to have minimal daily reminders of ITP and are interested in a therapy with high probability of platelet count response who have low risk with undergoing surgical procedure may be good candidates for splenectomy; alternatively, patients who have failed or are not good candidates for medical therapy may have few other options.

\section{Rituximab}

As rates of splenectomy declined, use of rituximab increased and initial response rates were promising. Several studies examining the efficacy of rituximab as an alternative to splenectomy in patients with ITP, using the standard dosing of $375 \mathrm{mg} / \mathrm{m}^{2} /$ dose $\times 4$ doses, resulted in initial response rates of 40 to $60 \% .{ }^{19}$ Unfortunately, the long-term response rates with rituximab are not as good as splenectomy with sustained response of approximately $20 \%$ at 5 years postinitial rituximab treatment. ${ }^{20} \mathrm{~A}$ recent trial in 112 adult patients comparing standard dosing of rituximab and placebo showed no difference in complete response (CR) at 1.5 years. ${ }^{21}$ Many patients who initially respond to rituximab can respond to subsequent doses; however, the safety and efficacy of repeated dosing of rituximab has not been systematically evaluated.

In addition, rituximab is associated with significant side effects. Approximately $1 \%$ of patients treated are unable to complete a full course because of infusion reactions, while 1 to $2 \%$ of patients develop persistent hypogammaglobulinemia post-therapy, which can be associated with infection and development of common variable immunodeficiency. ${ }^{21}$ For this reason, it is important to check immunoglobulin levels and B cell recovery after rituximab therapy. Some guidelines recommend following similar practices prior to rituximab therapy as for splenectomy. ${ }^{22}$

Efforts to reduce side effects, but maintain efficacy, have resulted in various dosing regimens that have been used in treatment of ITP including low-dose regimens $\left(100 \mathrm{mg} / \mathrm{m}^{2}\right.$ weekly or $100 \mathrm{mg}$ weekly) that have been used alone $\mathrm{e}^{23,24}$ or in combination with other medications such as dexamethasone and cyclosporine ${ }^{25}$ or recombinant human thrombopoietin. ${ }^{26}$

\section{Other Immunomodulatory Therapies}

A recent (2017) systematic review examined the literature published in English for studies using several immunomodulatory treatments that have been reported in clinical studies in small numbers of patients. ${ }^{27}$ Some of these options, particularly dapsone and azathioprine, are especially useful in countries where access to some of the more expensive medications such as TPO-RA and rituximab is more limited. Some of the immunomodulatory medications were covered in this systematic review, but others have been used, and the limited data available are presented in -Table 1 .

\section{Dapsone}

Dapsone has been studied in both adults and children with persistent and chronic ITP. Approximately 80 adult patients were evaluated prospectively (reviewed in Weber et al. ${ }^{27}$ ) 


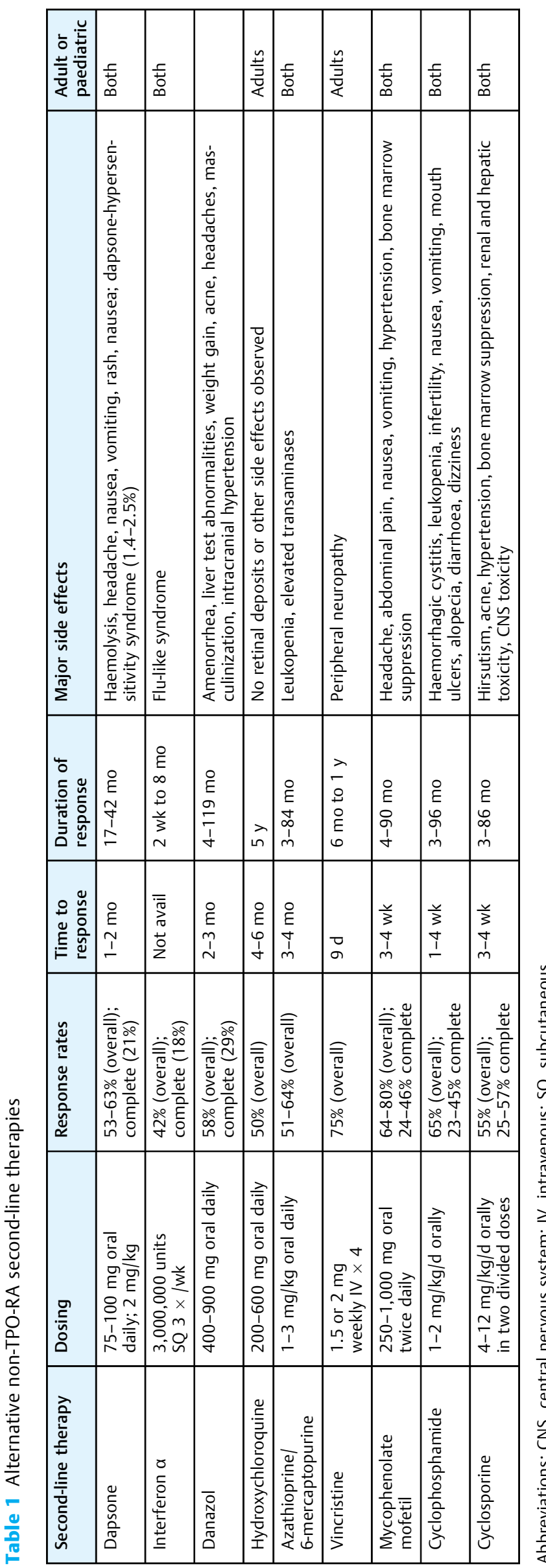

with an additional 141 patients (both adults and children) reported by retrospective review of medical records after treatment. ${ }^{28-30}$ Dosing is generally 75 to $100 \mathrm{mg}$ daily for adults and $2 \mathrm{mg} / \mathrm{kg}$ in paediatric patients. In both groups of patients, response rates were 53 to $63 \%$ (except the smallest retrospective study, which had a response rate of only $11 \%$ in nine patients ${ }^{28}$ ). Time to response is relatively slow with most patients requiring 1 to 2 months to respond. Duration of response ranged from 17 to 42 months, but responses generally require ongoing therapy. ${ }^{27}$

Side effects are relatively common and included haemolysis without anaemia or methemoglobinemia, haemolytic anaemia, as well as headache, nausea, vomiting and rash. ${ }^{27}$ Severe dapsone-induced hypersensitivity syndrome is rare, characterized by generalized skin eruption with one or more of the following: (1) fever, (2) lymphadenopathy or (3) hepatis, and occurs in approximately $1.4 \%$ of patients. ${ }^{31}$ This reaction may be associated with the HLA-B*13:01 polymorphism among patients of Indian origin (at least in those receiving dapsone for leprosy). ${ }^{32}$

\section{Azathioprine/6-Mercaptopurine}

Purine anti-metabolites, azathioprine and 6-mercaptopurine (6MP), were developed as a chemotherapeutic in the 1950s. Azathioprine is slowly but completely metabolized to $6 \mathrm{MP}$ and is felt, generally, to have fewer side effects. As suppressors of both $\mathrm{B}$ and $\mathrm{T}$ lymphocytes, they have been used in both autoimmune haemolytic anaemia and ITP since the 1960s. ${ }^{33}$ The anti-metabolites have been used in small series and at least two larger adult series and one paediatric trial demonstrating response rates of approximately 51 to $64 \%$ (up to $87 \%$ in smaller series and $83 \%$ for $6 \mathrm{MP}$ in paediatrics ${ }^{34}$ ). Most commonly reported side effects include leukopenia (and less commonly other cytopenias due to bone marrow suppression) and elevated transaminases ( $3 \times$ upper limit of normal, ULN). Careful attention should be paid to concomitant medications, as several drug-drug interactions can be important. Time to response ranged from 0.7 to 11 months (with a median of 3-4 months) 35 and duration of response was from 3 to 84 months. ${ }^{35}$

\section{Cyclophosphamide}

Cyclophosphamide has been used primarily as an adjunct in severe, refractory ITP. First reported in 1971, cyclophosphamide is one of the oldest adjunctive immunosuppressive therapies that have been used. ${ }^{36}$ Severe toxicities, such as haemorrhagic cystitis, leukopenia and risk of secondary malignancies limit its routine use (although these are more common with the intravenous dosing). Long-term use may compromise fertility. Even the common toxicities (nausea, vomiting, mucosal ulcers, alopecia, diarrhoea and dizziness) are more onerous than many of the other currently available options, making cyclophosphamide an option for patients with truly refractory disease that fails to respond to other less toxic therapies. Response rates are similar to other treatments with overall response rates of approximately $65 \%$ and CR of 23 to $45 \%$ with partial response (PR) of $20 \% .{ }^{37}$ The dose most commonly used is 1 to $2 \mathrm{mg} / \mathrm{kg} / \mathrm{day}$ (generally 
approximately $100-200 \mathrm{mg}$ for an adult patient) and the drug should be administered after platelet count response for an additional 2 to 3 months and then discontinued. ${ }^{37}$ Responses are usually within 2 to 10 weeks after initiation of therapy ${ }^{36}$ inducing remissions lasting up to 96 months.

\section{Cyclosporin}

Cyclosporin, a calcineurin inhibitor, inhibits production of interleukin 2, which leads to an inhibition of T cell activation. This then suppresses T-cells and modulates the immune response and can lead to improved platelet counts in some patients with ITP. Reponses typically occur in approximately 4 weeks when cyclosporine is administered orally at 4 to 12 $\mathrm{mg} / \mathrm{kg} /$ day in two divided doses. Side effects include hirsutism and acne, which may be quite distressing to patients. In addition, hypertension, renal insufficiency and hepatotoxicity can be seen and careful monitoring is needed. ${ }^{38}$ Finally, patients may also develop myelosuppression and neurologic complications including tremors and seizures (usually due to posterior reversible encephalopathy syndrome, a complication of hypertension). Overall responses, however, are similar to most other second-line therapies for chronic ITP at approximately $55 \%$ with PR of 8 to $30 \%$ and CR of 57 to $25 \%{ }^{39-41}$

\section{Mycophenolate Mofetil}

Mycophenolate mofetil (MMF) was first reported as a potential therapy for steroid-resistant ITP in $2002 .{ }^{42}$ MMF works by suppression of $\mathrm{T}$ cells, similar to azathioprine and cyclosporine, but by inhibition of inosine-5'-monophosphate dehydrogenase (IMPDH), a key enzyme in the purine biosynthesis pathway resulting in adenine accumulation and relative guanine deficiency ultimately causing cell cycle arrest. Because lymphocytes are dependent upon purine synthesis due to inability to recycle purine nucleotides and because some $\mathrm{T}$ cell activation steps are guanosine triphosphate dependent, MMF inhibits T cell activation and proliferation. Since the first report, others have reported on the use of MMF as monotherapy or in combination with corticosteroids for ITP with overall response rates as high as 64 to $80 \% 38,43-46$ and CR of 24 to $46 \%$ and PR of 18 to $29 \%$. Patients received between 250 and 1,000 mg twice daily (total dose: 2 $\mathrm{g} /$ day). Generally, responses are somewhat delayed, as with some of the other immunosuppressive therapies, taking 3 to 4 weeks to see a response in most patients. Common toxicities include headache, abdominal pain, nausea and vomiting. Hypertension may also be seen and patients should have regular blood pressure monitoring. Finally, because of the effect on purine metabolism, other cytopenias may result as a result of bone marrow suppression (leukopenia, anaemia, and thrombocytopenia). ${ }^{38}$

\section{Other Therapies}

Several other immunomodulatory therapies have been used alone or in combination with other treatments as second- or third-line treatment for ITP either after relapse post-splenectomy or in an effort to avoid splenectomy. Some of these therapies are listed in -Table 1. The majority of these treatments have shown some efficacy in some patient popu- lations. The difficulty is to identify the appropriate patient who is going to respond to any given therapy. This is often a process of trial and observation and can be frustrating for both patient and clinician as they embark on the next treatment. Novel immunomodulatory therapies, including the newly approved spleen tyrosine kinase (Syk) inhibitor, fostamatinib, also demonstrate efficacy in some patients. This medication, which is expected to disrupt Fc receptor signalling and phagocytosis of antibody-coated platelets, resulted in 37 to $48 \%$ overall response rates in two recently published phase III clinical trials of adult patients with highly refractory chronic ITP, with stable response rates of $18 \%$ (vs. $2 \%$ in placebo; $p<0.001$ ). ${ }^{47,48}$ Side effects included diarrhoea and hypertension requiring monitoring and potentially dose reduction. Additional novel therapies including an anti-neonatal Fc receptor (FcRn) agent (rozanolixizumab) which prevents recycling of the FcRn and may reduce the half-life of circulating anti-platelet antibodies is currently in phase II trials in patients with ITP. ${ }^{49,50}$ Monoclonal antibodies targeting CD154 and CD40 to target the interactions of T cells with $B$ cells and prevent the development of autoreactive $T$ cells/B cell populations are also in development and in clinical trials, although enthusiasm has been tempered somewhat by increased risk of thrombosis in early trials.

\section{Summary}

In summary, ITP is a complex disease. Second-line therapy for ITP conventionally consists of splenectomy, rituximab or oral immunomodulatory therapy. TPO-RAs have begun to play a major role in second-line therapy (discussed elsewhere in this issue) and direct comparison of outcomes has yet to be performed to establish a true hierarchy to guide clinical management. In the absence of evidence, patient and clinician must establish mutual goals of treatment to optimally determine therapy, and not all patients will choose medical therapy over surgical intervention. Novel therapies are in development and may further shift the current practice from splenectomy, but data comparing outcomes are still lacking.

\section{Authorship and Conflict of Interest}

M.P.L. drafted the manuscript and provided revisions. She has received honoraria and provided consultancy to Amgen, Novartis, Shionogi, Sysmex, Educational Concepts in Medicine, DynaMed, and Bayer. She has received research funding from Astra Zeneca, Inc.

\section{References}

1 Moulis G, Palmaro A, Montastruc JL, Godeau B, Lapeyre-Mestre M, Sailler L. Epidemiology of incident immune thrombocytopenia: a nationwide population-based study in France. Blood 2014;124 (22):3308-3315

2 Fogarty PF, Segal JB. The epidemiology of immune thrombocytopenic purpura. Curr Opin Hematol 2007;14(05):515-519

3 Schifferli A, Holbro A, Chitlur M, et al; Intercontinental Cooperative ITP Study Group (ICIS). A comparative prospective observational study of children and adults with immune thrombocytopenia: 2-year follow-up. Am J Hematol 2018;93(06):751-759 
4 Neunert C, Lim W, Crowther M, Cohen A, Solberg L Jr, Crowther MA; American Society of Hematology. The American Society of Hematology 2011 evidence-based practice guideline for immune thrombocytopenia. Blood 2011;117(16):4190-4207

5 Rodeghiero F, Stasi R, Gernsheimer T, et al. Standardization of terminology, definitions and outcome criteria in immune thrombocytopenic purpura of adults and children: report from an international working group. Blood 2009;113(11):2386-2393

6 Chaturvedi S, Arnold DM, McCrae KR. Splenectomy for immune thrombocytopenia: down but not out. Blood 2018;131(11): $1172-1182$

7 Tamary H, Roganovic J, Chitlur M, Nugent DJ. Consensus PaperICIS Expert Meeting Basel 2009 treatment milestones in immune thrombocytopenia. Ann Hematol 2010;89(Suppl 1):5-10

8 Kaznelson P. Verschwinden der hamorrhagischen Diathese bei einem Fane von "essentieller Thrombopenie" (Frank) nach Milzextirpation. Klin Wochenschr 1916;29:1451-1454

9 Kojouri K, Vesely SK, Terrell DR, George JN. Splenectomy for adult patients with idiopathic thrombocytopenic purpura: a systematic review to assess long-term platelet count responses, prediction of response, and surgical complications. Blood 2004;104(09): 2623-2634

10 Gwilliam NR, Lazar DA, Brandt ML, et al. An analysis of outcomes and treatment costs for children undergoing splenectomy for chronic immune thrombocytopenia purpura. J Pediatr Surg 2012; 47(08):1537-1541

11 Palandri F, Polverelli N, Sollazzo D, et al. Have splenectomy rate and main outcomes of ITP changed after the introduction of new treatments? A monocentric study in the outpatient setting during 35 years. Am J Hematol 2016;91(04):E267-E272

12 Kumar S, Diehn FE, Gertz MA, Tefferi A. Splenectomy for immune thrombocytopenic purpura: long-term results and treatment of postsplenectomy relapses. Ann Hematol 2002;81(06):312-319

13 Ahmed R, Devasia AJ, Viswabandya A, et al. Long-term outcome following splenectomy for chronic and persistent immune thrombocytopenia (ITP) in adults and children : Splenectomy in ITP. Ann Hematol 2016;95(09):1429-1434

14 Thai LH, Mahévas M, Roudot-Thoraval F, et al. Long-term complications of splenectomy in adult immune thrombocytopenia. Medicine (Baltimore) 2016;95(48):e5098

15 Guan Y, Wang S, Xue F, et al. Long-term results of splenectomy in adult chronic immune thrombocytopenia. Eur J Haematol 2017; 98(03):235-241

16 Shatz DV. Vaccination considerations in the asplenic patient. Expert Rev Vaccines 2005;4(01):27-34

17 Rodeghiero F. A critical appraisal of the evidence for the role of splenectomy in adults and children with ITP. Br J Haematol 2018; 181(02):183-195

18 Wang KK, Charles C, Heddle NM, Arnold E, Molnar L, Arnold DM. Understanding why patients with immune thrombocytopenia are deeply divided on splenectomy. Health Expect 2014;17(06): 809-817

19 Cuker A, Cines DB, Neunert CE. Controversies in the treatment of immune thrombocytopenia. Curr Opin Hematol 2016;23(05): 479-485

20 Chugh S, Darvish-Kazem S, Lim W, et al. Rituximab plus standard of care for treatment of primary immune thrombocytopenia: a systematic review and meta-analysis. Lancet Haematol 2015;2 (02):e75-e81

21 Ghanima W, Khelif A, Waage A, et al; RITP study group. Rituximab as second-line treatment for adult immune thrombocytopenia (the RITP trial): a multicentre, randomised, double-blind, placebo-controlled trial. Lancet 2015;385(9978):1653-1661

22 Moulis G, Lapeyre-Mestre M, Mahévas M, Montastruc JL, Sailler L. Need for an improved vaccination rate in primary immune thrombocytopenia patients exposed to rituximab or splenectomy. A nationwide population-based study in France. Am J Hematol 2015;90(04):301-305
23 Kapoor R, Kumar R, Mahapatra M, Pati HP, Pramanik SK. Low dose rituximab in chronic ITP: still an option in resource limited settings. Indian J Hematol Blood Transfus 2017;33(04):568-573

24 Tran H, Brighton T, Grigg A, et al. A multi-centre, single-arm, open-label study evaluating the safety and efficacy of fixed dose rituximab in patients with refractory, relapsed or chronic idiopathic thrombocytopenic purpura (R-ITP1000 study). Br J Haematol 2014;167(02):243-251

25 Choi PY, Roncolato F, Badoux X, Ramanathan S, Ho SJ, Chong BH. A novel triple therapy for ITP using high-dose dexamethasone, low-dose rituximab, and cyclosporine (TT4). Blood 2015;126(04): 500-503

26 Li Y, Wang YY, Fei HR, Wang L, Yuan CL. Efficacy of low-dose rituximab in combination with recombinant human thrombopoietin in treating ITP. Eur Rev Med Pharmacol Sci 2015;19(09): $1583-1588$

27 Weber E, Reynaud Q, Fort R, et al. Immunomodulatory treatments for persistent and chronic immune thrombocytopenic purpura: a PRISMA-compliant systematic review and meta-analysis of 28 studies. Medicine (Baltimore) 2017;96(37):e7534

28 Lee JY, Lee JO, Jung JY, Bang SM. Dapsone therapy for refractory immune thrombocytopenia patients: a case series. Blood Res 2017;52(02):95-99

29 Estève C, Samson M, Guilhem A, et al. Efficacy and safety of dapsone as second line therapy for adult immune thrombocytopenia: a retrospective study of 42 patients. PLoS One 2017;12 (10): $\mathrm{e} 0187296$

30 Damodar S, Viswabandya A, George B, Mathews V, Chandy M, Srivastava A. Dapsone for chronic idiopathic thrombocytopenic purpura in children and adults-a report on 90 patients. Eur J Haematol 2005;75(04):328-331

31 Sauvetre G, MahÉvas M, Limal N, et al. Cutaneous rash and dapsone-induced hypersensitivity syndrome a common manifestation in adult immune thrombocytopenia. Presentation and outcome in 16 cases. Am J Hematol 2015;90(10):E201-E202

32 Zhang FR, Liu H, Irwanto A, et al. HLA-B*13:01 and the dapsone hypersensitivity syndrome. N Engl J Med 2013;369(17): $1620-1628$

33 Schwartz R, Dameshek W. The treatment of autoimmune hemolytic anemia with 6-mercaptopurine and thioguanine. Blood 1962;19:483-500

34 Sobota A, Neufeld EJ, Lapsia S, Bennett CM. Response to mercaptopurine for refractory autoimmune cytopenias in children. Pediatr Blood Cancer 2009;52(01):80-84

35 Quiquandon I, Fenaux P, Caulier MT, Pagniez D, Huart JJ, Bauters F. Re-evaluation of the role of azathioprine in the treatment of adult chronic idiopathic thrombocytopenic purpura: a report on 53 cases. Br J Haematol 1990;74(02):223-228

36 Verlin M, Laros RK Jr, Penner JA. Treatment of refractory thrombocytopenic purpura with cyclophosphamine. Am J Hematol 1976;1(01):97-104

37 Berchtold P, McMillan R. Therapy of chronic idiopathic thrombocytopenic purpura in adults. Blood 1989;74(07):2309-2317

38 Provan D, Moss AJ, Newland AC, Bussel JB. Efficacy of mycophenolate mofetil as single-agent therapy for refractory immune thrombocytopenic purpura. Am J Hematol 2006;81(01):19-25

39 Emilia G, Morselli M, Luppi M, et al. Long-term salvage therapy with cyclosporin A in refractory idiopathic thrombocytopenic purpura. Blood 2002;99(04):1482-1485

40 Kappers-Klunne MC, van't Veer MB. Cyclosporin A for the treatment of patients with chronic idiopathic thrombocytopenic purpura refractory to corticosteroids or splenectomy. $\mathrm{Br} \mathrm{J}$ Haematol 2001;114(01):121-125

41 Moskowitz IP, Gaynon PS, Shahidi NT, Cripe TP. Low-dose cyclosporin A therapy in children with refractory immune thrombocytopenic purpura. J Pediatr Hematol Oncol 1999;21(01):77-79

42 Howard J, Hoffbrand AV, Prentice HG, Mehta A. Mycophenolate mofetil for the treatment of refractory auto-immune haemolytic 
anaemia and auto-immune thrombocytopenia purpura. $\mathrm{Br}$ Haematol 2002;117(03):712-715

43 Hou M, Peng J, Shi Y, et al. Mycophenolate mofetil (MMF) for the treatment of steroid-resistant idiopathic thrombocytopenic purpura. Eur J Haematol 2003;70(06):353-357

44 Zhang WG, Ji L, Cao XM, et al. Mycophenolate mofetil as a treatment for refractory idiopathic thrombocytopenic purpura. Acta Pharmacol Sin 2005;26(05):598-602

45 Taylor A, Neave L, Solanki S, et al. Mycophenolate mofetil therapy for severe immune thrombocytopenia. Br J Haematol 2015;171 (04):625-630

46 Miano M, Ramenghi U, Russo G, et al. Mycophenolate mofetil for the treatment of children with immune thrombocytopenia and Evans syndrome. A retrospective data review from the Italian association of paediatric haematology/oncology. Br J Haematol 2016;175(03):490-495
47 Bussel J, Arnold DM, Grossbard E, et al. Fostamatinib for the treatment of adult persistent and chronic immune thrombocytopenia: results of two phase 3 , randomized, placebo-controlled trials. Am J Hematol 2018;93(07):921-930

48 Newland A, Lee EJ, McDonald V, Bussel JB. Fostamatinib for persistent/chronic adult immune thrombocytopenia. Immunotherapy 2018;10(01):9-25

49 Erratum for the Research Article. Erratum for the research article: "The FcRn inhibitor rozanolixizumab reduces human serum IgG concentration: a randomized phase 1 study" by P. Kiessling, R. Lledo-Garcia, S. Watanabe, G. Langdon, D. Tran, M. Bari, L. Christodoulou, E. Jones, G. Price, B. Smith, F. Brennan, I. White, S. Jolles. Sci Transl Med 2017;9(419):eaar6448

50 Kiessling P, Lledo-Garcia R, Watanabe S, et al. The FcRn inhibitor rozanolixizumab reduces human serum $\operatorname{IgG}$ concentration: a randomized phase 1 study. Sci Transl Med 2017;9(414):eaan1208 